\title{
Neurodynamic Models of Brain in Psychiatry
}

\author{
Walter J Freeman*,I \\ 'Department of Molecular \& Cell Biology, University of California, Berkeley, CA, USA
}

The history of brain theory is described in terms of three kinds of theory of perception. The most widely used kind sees perception as dependent on passive inflow from the environment of information that is used to make and process representations of objects and events. A second kind views perception as an active search for information that is inherent in the environment and is extracted by tuned resonances in brain circuits. A third kind holds that perception works by the creation of information through chaotic dynamics by forming hypotheses about the environment, through which learning takes place. Experimental evidence for creative dynamics in brains is briefly sketched. The explanation is offered that brains, being finite systems, work this way in order to cope with the infinite complexity of the world. All that brains can know is the hypotheses they construct and the results of testing them by acting into the environment, and learning by assimilation from the sensory consequences of their actions. The process is described as intentionality. It works through the action-perception-assimilation cycle. The cost of this solution to the problem of infinite complexity by hypothesis testing is the progressive isolation of individuals, as they accumulate their unique experiences through which their personalities form. Socialization and the acquisition of shared knowledge requires the emergence of new personality structure by self-organization through chaotic dissolution of existing the structure, as a prelude to the creation of new traits, habits, and values. Dissolution works in a crisis situation by regression to earlier stages of development, from which a fresh start can be made. A state of malleability emerges in the depth of crisis, in which compassionate companions through loving care can invite cooperative actions. Joint actions support the growth of a new lifestyle based on trust. Socialization requires neurochemical mechanisms of affiliation and bonding that evolved through the requirements of parental care of altricial offspring in mammalian reproduction. These mechanisms are invoked by means of behavioral techniques from cultural evolution. The dynamics, neural mechanisms, behavioral signs, methods of induction, and therapeutic utility of dissolution should be known by therapists. Lack of recognition and understanding may cause failure to use brief windows of opportunity to instill long-term relief of psychic pain by restructuring intentionality in distressed patients.

Neuropsychopharmacology (2003) 28, S54-S63. doi:I0.1038/s..npp. 1300147

Keywords: assimilation; brain theory; intentionality; neurodynamics; psychodynamics

\section{INTRODUCTION: A BRIEF REVIEW OF THE HISTOR- ICAL EMERGENCE OF BRAIN THEORY}

Psychiatry has two deep roots, one in the experiences people have through living together in social groups, the other in the experiences people have through interacting with the material world. Both kinds of experience are sifted through private reflection and integration. Many centuries of systematic experimentation and synthesis have fostered the growth of bodies of knowledge about these experiences that comprise the social and natural sciences. Health practitioners draw inferences from this knowledge to offer treatment regimens for individuals who are experiencing distress and disorder beyond their competence for achieving or restoring the desired comfort in their lives. The

*Correspondence: Dr WJ Freeman, Department of Molecular \& Cell Biology, University of California, Berkeley, CA 94720-3200, USA, Tel: + | 510642 4220, Fax: + | 510643 679|,

E-mail: wfreeman@socrates.berkeley.edu, http://sulcus.berkeley.edu Received 0I September 2002; revised 03 November 2002; accepted 03 December 2002 regimens that can be inferred from these distinctive bodies of knowledge are inherently disjunct and may often be in opposition. Physicians have particular difficulty in adjudicating conflicting claims for efficacy of particular regimens deriving from these alternative sources. For example, until the early 19th century, physicians held with Hippocrites that mental disorders stemmed from imbalances in the four humors of the body, while priests preached the wages of sin, and astrologers looked to malign stars. If personal income was any measure, those who cast horoscopes and sold indulgences were far more successful in mental health care than were the physicians and surgeons of those millennia.

These standings began to change in the middle of the 19th century with the explosive growth in physics, chemistry, and biology. Psychiatrists, pre-eminently Bleuler and Charcot, increasingly looked for material causes of mental disorders such as schizophrenia and hysteria. Biologists were spectacularly successful in identifying Treponema pallidum as the cause of general paresis and salvarsan as the therapeutic 'magic bullet', thereby creating a compelling biological model of mental disorders for a new generation of 
practitioners. What remained after other successes in assigning material causes of aberrant behavior to viruses (rabies, polio, measles), environmental toxins (lead, hydrocarbons, ergot), vitamin and mineral deficiencies (cretinism, pellagra, beri beri), hormonal deficits (hypothyroidism, diabetic coma, lack of dopamine in postencephalitic and idiopathic Parkinson's disease), and genetic abnormalities (phenylketonuria, Tourette's syndrome, Huntingdon's chorea) came to be known as 'functional disorders', which opened the door to brain theory and the concepts of what Geschwind called 'deconnection' disorders (Geschwind and Kaplan, 1962) and Glass and Mackey (1988) called 'dynamic diseases'.

The core concept of brain theory in the 19th century was nerve energy, and the parent science was thermodynamics. The flow of nerve energy was conceived to follow hierarchies of reflexes along axonal pathways and through 'contact barriers' between neurons that offered resistance. Giving due place to the first law of thermodynamics, nerve energy was conceived to be conserved, so that if blocked by high resistance in one path, the energy would take another. Freud (1895) in his attempt to lay a scientific foundation for psychoanalysis, later abandoned as 'premature', labelled nerve energy as ' $Q$ ' and wrote:

'This line of approach is derived directly from pathological clinical observations, especially those concerned with excessively intense ideas. These occur in hysteria and obsessional neurosis, where, as we shall see, the quantitative characteristic emerges more plainly than in the normal. What I have in mind is the principle of neuronic inertia, which asserts that neurones tend to divest themselves of quantity (Q). We arrive at the idea of a 'cathected' neurone (N) filled with a certain quantity. The principle of inertia finds expression in the hypothesis of a current, passing from the cell-processes or dendrites to the axone. The secondary function [memory] is made possible by supposing that there are resistances which oppose discharge. in the contacts [between the neurones] which thus function as barriers. The hypothesis of 'contact-barriers' is fruitful in many directions.

Indeed that was an understatement, in that the greater part of biological psychiatry is now based on synaptic transmitter chemistry.

In the 20th century the core concept of nerve energy was replaced by 'information', which was carried by action potentials and processed in the networks of neurons in accordance with the parent science of information theory. This model has not been well received by psychiatrists, because the basis in logic neglects the emotional, irrational, and chaotic formants of thinking and behavior. The limitations of information processing have become ever more apparent with the growth of neural networks, which require that the dynamics of neurons and networks be frozen into frames, discretized into rational numbers, and linearized in order to use matrix algebra to describe operations on input vectors. Moreover, in the same century the growth of empirical neurochemistry and neuropharmacology has given psychiatrists an array of powerful neuroactive and psychoactive drugs with which to treat mental diseases. Unfortunately, these empirical remedies are accompanied neither by sound theoretical explanations of their actions nor by optimal schedules of administration.
Practitioners are currently making do by trial and error under heavy pressures from health maintenance organizations to get the patients out the door, while differences between diverse accounts of the social and biological sources, and dimensions of the pathogenesis and treatment of functional mental disorders remain unresolved.

Brain theory continues to grow explosively, fed by new data from the basic sciences and clinical uses of brain imaging, and by the parent sciences of complexity and nonlinear dynamics. These sciences are quite new entries into the situation, so it is premature to evaluate their utility for clinical judgment and selection of treatment regimens. They can provide valuable insights into the nature of brain function, how information theory can be applied, and the ways in which nonlinear dynamics may be used to construct new and more powerful brain theory. My approach in this essay is to review the platforms on which the cognitive sciences are based, to explain briefly some new observations on brain function that have been guided by nonlinear dynamics, to describe some implications for brain theory, and from these insights to offer some suggestions regarding how the relations between psychiatrists and psychoanalysts and their patients or clients might be reinterpreted and clarified to the benefit of all.

\section{AN OVERVIEW OF THE COGNITIVE SCIENCES}

I begin with an overview of the cognitive sciences. These sciences are broadly concerned with deriving the rules by which information from the world is gathered through the senses, processed in the brain, integrated, stored, retrieved, and deployed through muscular actions. There are many pathways that are being followed in this research. Neurologists observe and treat the disorders of thinking and of behavior in patients with brain damage, disease, and unusual developmental outcomes such as autism. Neurobiologists examine the brains and behaviors of animals using electrophysiological and imaging techniques. Psychologists investigate the stages by which infants and children develop competence in dealing with their unfolding worlds. Philosophers sift through the great systems of thought that have been distilled from human experience. Computer scientists model logic and language. Mathematicians design systems and computer-based dynamical devices that simulate and emulate thought processes, as we experience them through logic, introspection, and phenomenology. Owing to my predominant concern for the relations between cognition and behavior, I emphasize those aspects that are described as 'embodied cognition', in which the body serves as the principal tool of the brain for cognitive development and the acquisition of knowledge, and as 'situated cognition', in which the structure of the environment determines, and is determined by, the actions of individuals seeking knowledge. A truism for most experimental scientists is that knowledge of the material world comes through the interface provided by the senses between the world and the brain. This view stands in opposition to alternative views that knowledge is implanted in the genome by evolution and is revealed in the course of ontogenesis to the individual and others, or that knowledge is received through immaterial intervention that is experi- 
enced by the individual as inspiration or revelation. I state this truism at the outset to clarify my premise that all knowledge gained by individuals is earned by their actions into the world, from which they suffer the due consequences. The goal of the neurocognitive scientist is to describe, in terms of brain dynamics, how these actions are conceived, planned, and executed in the brains of individuals.

Since the ancient Greeks, scientists have held two conceptions about how our brains work to understand their environments. Scientists working in the tradition of Plato conceive perception as passive. The classic metaphor is Plato's cave, in which light coming from outside cast shadows on the walls and entered the eyes bearing indistinct and incomplete forms. These forms were compared by reason with a store of ideal forms for identification in an operation we now call 'pattern recognition'. Actions were selected by ethical judgment using the moral faculty, now being replaced by game theory. In modern terms, this view is described as information processing by the brain using hierarchies of reflex arcs and neural networks. Action begins with the senses, particularly the eyes and skin, when neurons respond to patterns of energy, such as textures of light and dark, of smoothness and edges, and generate patterns of action potentials that carry the forms of objects. Thus, the sense organs encode the forms input as information. The information is carried from the sense organs by action potentials through relays into the upper levels of the brain. There the information is refined in the sensory cortices, stored in the frontal lobes, retrieved, and compared with new information so as to classify fresh stimuli and select appropriate emotions and courses of action. Actions and emotions are selected from menus of algorithms stored in the amygdala and basal ganglia. Movements are initiated by motor commands that descend into the brain stem and spinal cord to contract the muscles in response to the inputs. Behaviorism to the extent that it concerns brains at all (Skinner, 1969), 'strict AI' (artificial intelligence), industrial robotics, and feedforward neural networks using supervised learning are all based on this Platonic model.

The alternative classic view, propounded by the Aristotelians, is that perception is active. It requires movement into the world by probing, cutting, and burning in order to learn by manipulation the forms, textures, weights, and appearances of objects. Behavior is proactive, not reactive. This model is central to a variety of cognitive systems such as pragmatism, existentialism, Piagetian developmental psychology, Gestaltism, its derivative, ecological psychology, and embodied cognition. In these modern views, information is implicit in the objects that the brain is seeking. The search is initiated when the brain creates a goal with a need for information to realize that goal, and the brain directs the sense organs to find the information in the world, using the cognitive map in the medial temporal lobe to direct the search. At the same time the brain prepares the sensory circuits by tuning them with copies of the motor commands called 'corollary discharges' (Sperry, 1950; Freeman, 2001) that selectively sensitize the cortices to the desired input. The information carried by objects is detected by the senses. They send it to the sensory cortices where it is extracted by resonances in the neural circuits that are tuned just before the search takes place. These actions and the prior tuning constitute the exercise of foresight and selective attention. They limit the entry to the desired information, not whatever forms or energies from irrelevant objects happen to enter the sense organs or be put there by a naive experimenter. A key type of information selected by the resonances corresponds to the uses to which the objects are to be put, their 'affordances' (Gibson, 1979). This also is the domain of recurrent neural networks using unsupervised learning, which is the blind probing for structure that extracts information by gradient descent in high-dimensional measurement spaces.

A third view differs from both these classic views. This approach was pioneered by Saint Thomas Aquinas (1272) in his effort to bring Aristotelian doctrine into conformance with Christianity. The basic Thomist premise is the unity and inviolability of the self that is inherent in the soul, brain, and body. This unity does not allow the entry of forms (information) into the self. The impact of the world onto the senses gives rise to states of activity he called 'phantasms', which are ephemeral and unique to each impact and therefore cannot be known. The function of the brain is to exercise the faculty of the imagination, which is not present in the Aristotelian view, in order to abstract and generalize over the phantasms that are triggered by unique events. These processes of abstraction and generalization create information that assimilates the body and brain to the world. Assimilation is not adaptation by passive information processing, nor is it an accumulation of representations by resonances. It is the shaping of the self to bring it into optimal interaction with the desired aspects of the world. The goal of an action is a state of competence that Maurice Merleau-Ponty (1945) called 'maximum grip'. Assimilation is the beginning for all knowledge.

Thus, the manner of acquisition of knowledge is by thrusting the body into the world, from which our word 'intention' has come from the Latin 'intendere' = 'stretching forth'. The thrust initiates the action-perception cycle, which is followed by the changes through which the self learns about the world, and ultimately about God, by assimilation (from the Latin 'adequatio' = adequacy) of the self to the world. There is no transfer of information across the senses into the brain, but instead the creation of information within the brain under the existing constraints of the brain and body. In this respect cognition is related to digestion, which protects the integrity of the immunological self by breaking all forms of foodstuffs into elementary ions and molecules that are absorbed and built into complex macromolecules, each now bearing the immunological signature of the individual self. Similarly, events and objects in the world are broken into sheets of action potentials like pinpoints of light, the 'raw sense data' of analytic philosophers and the phantasms of Thomists, and new forms emerge through constructions by the chaotic dynamics in sensory cortices. The explanation for this manner of function of both the neural and the digestive systems is essentially the same: the world is infinitely complex, and the self can only know and incorporate what the brain makes within itself. This is why neurobiologists using passive neural networks cannot solve the figureground problem, why linguists cannot do machine translation, why philosophers cannot solve the symbol-grounding 
problem, why cognitive scientists cannot surmount the limitations of expert systems, and why engineers cannot yet build autonomous robots capable of operating in unstructured environments. The unbounded complexity of the world defeats those classic Platonic and Aristotelian approaches.

\section{THE NEURAL DYNAMICS OF THE CONSTRUCTION OF INDIVIDUAL EXPERIENCE}

The Thomist concept of intentionality offers a way to solve these intractable problems, because they stem largely from the attempt by Descartes to make mathematics the foundation of the natural sciences instead of their principal tool for quantitative analysis. Thomist philosophy in the 13th century provided the basis for the explosive growth of medieval science, medicine, law, industry and navigation that nurtured the worldwide expansion of Western culture and the Renaissance, but Thomist philosophy was replaced by the Cartesian revolution in the 17th century, giving rise to modern science. If the current impasse is owing to Cartesian philosophy, then its predecessor, Descartes' target of opportunity, gives a well-documented starting place for an anti-Cartesian revolution. In particular, the process of intentionality offers a firm base for interpreting recent neurobiological data, which can explain how and why all knowledge is constructed within brains and is not imported through the senses as the forms of objects and events or as information or representations about them.

The evidence on which this assertion is based has come from my experiments on animals that have been trained to respond to conditioned stimuli (Freeman, 2000, 2001). I record the neural responses to the stimuli at various stages of transmission in the olfactory, visual, auditory, and somatomotor systems as the action potentials elicited by the stimuli course through the brain to the sensory cortices and beyond. The essential finding, alike in all the sensory systems, is that the neural activity directly evoked by a stimulus, the raw sense data, the phantasm, that is observed in bursts of input-driven action potentials, serves to select a pattern of activity to be created in and by that sensory cortex to which the stimulus is directed. The pattern generated by the cortex is not a 'representation' of the stimulus but is instead a briefly sustained neural discharge that constitutes the significance and value of the stimulus for the animal. Such patterns depend on past learning about the stimulus that has been embedded in the modified synapses in cortical networks, so the patterns are unique to each individual and not specific for the stimuli by which their construction is triggered. The sensory cortices broadcast these spatial patterns, while the raw sense data, the phantasms, having done their work, are deleted - attenuated by spatial filtering. The broadcasts overlap in the medial temporal lobe where the patterns combine to form multisensory percepts. The combined patterns are integrated into recent memory and located in environmental space by passage through the hippocampus with the cognitive map, and at this stage they are finally accessible to awareness as Gestalts.

Each cortical pattern resembles a visual picture in gray tones, in having a common carrier wave (like light) that is modulated in amplitude (light and dark). Each pattern holds briefly and dissolves, making way for the next in a sequence like frames in a movie film. Every pattern is created as a 'wave packet' by a phase transition in neuron populations that resemble the transformation of a gas into a liquid, like water vapor into a rain drop. Clouds of action potentials like water molecules in steam condense into scintillating disk in the cortex about the size of a toenail. The origin of the spatial pattern carried by a wave packet lies in the remote past as well as the recent involvement of the animal with its environment. The events leading to the formation of a wave packet in sensory cortex begin with the emergence in the whole brain of a conception of a future state, whether desired or feared, that is embodied in a collection of wave packets. This collection evolves into a neural motor command, which is intended to move the animal in search of the sensory input that is needed to facilitate realization of the desired future state. That command is accompanied by its copies, the corollary discharges that are sent to all of the sensory systems where they selectively sensitize the cortical networks for the modality-specific sensory consequences of the intended action. This preparation involves attention as well as intention, which together yield the state of expectancy, which simultaneously is impending action and the tuning of the sensory cortices to what the animal is seeking by looking, listening, sniffing, and probing. The response to an expected stimulus can be said to pre-exist the arrival of the stimulus by the creation of a hypothesis that is to be tested by an act of observation and perception. The hypothesis is the state of selective sensitivity that has the potential for creating a wave packet. The dynamic process of creating a spatial pattern of the wave packet is a form of generalization that identifies the class to which the stimulus belongs, including its meaning. Abstraction takes place when the sensory-driven activity, the raw sense data, is removed during the transmission of the wave packet.

The organization of brain dynamics that supports this recursive process is schematized in Figure 1. At the core of the brain is the collection of structures comprising the medial temporal lobe in humans and the essential components of the limbic system in all mammals. The entorhinal cortex receives from all sensory areas through

DYNAMIC ARCHITECTURE OF THE LIMBIC SYSTEM

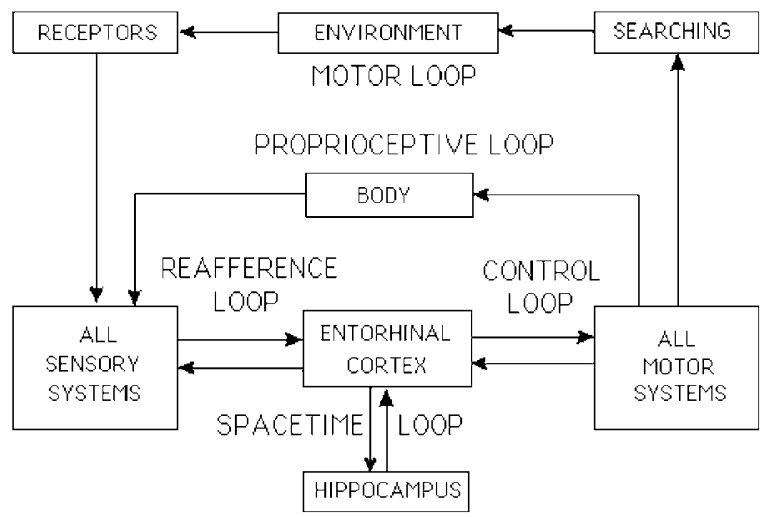

Figure I The process of intention is supported by multiple dynamic loops. 
multiple relays, such as the inferotemporal cortex in vision, the superior temporal gyrus in audition, the posterior cingulate in somesthesis, as well as the dorsal and ventral frontal lobes via the anterior cingulate and the uncinate fasciculus. Part of its output is sent to the amygdala and the corpus striatum in motor control, but the greater part is sent to the hippocampus, which establishes temporal and spatial orientation, and returns this information via the parasubiculum to the deeper layers of the entorhinal cortex. This is the 'spacetime loop'. The entorhinal cortex sends this information constituting corollary discharge to all sensory systems via the 'reafference loop', which provides predictions for the sensory changes accruing from motor actions. This loop is distinct from the several components within the brain such as the corticocerebellar connections that form the 'control loop', and the feedback through the body that comprises the 'proprioceptive loop', and the sensory feedback through the 'motor loop' into the environment.

The formation of ever-changing goal states and perceptual hypotheses requires the repeated construction of wave packets with novel spatial patterns. This construction is done by neural circuits that generate carrier waves, which continually vary in form and confer the property that each construction is never twice identical to those preceding (Freeman, 2000). The capability for such novelty resides in brain chaos. Chaotic dynamics has the property of creating information, which is essential for the construction of new goals that precede action, and new categories that precede perception. Chaos also has the capacity for destroying information, which is necessary for generalization and abstraction, and which is done by spatial and temporal filtering.

Every sensory cortex maintains a landscape of chaotic attractors that correspond to the perceptual categories that the subject is capable of discriminating (Ohl et al, 2001). When the subject attends to an expected event, the landscape is created, and a known stimulus gives access to the proper basin. After the dynamics converges to the selected attractor, the specific details of the stimulus are removed. The raw sense data by which category selection is performed are discarded after they are no longer useful. These properties of chaos are most in evidence when a hypothesis is disproved by testing, because the resulting stimulus is novel. Upon this failure, the prediction is changed by creative activity in the limbic system and disseminated by corollary discharges, so that a new hypothesis is tested. The process is repeated by the creation of new attractors by trial and error, until a reward is forthcoming and a hypothesis is proved.

The changes generated by chaotic dynamics are in the form of the modification of numerous synapses that interconnect the neurons in many parts of the brain. When the process finally succeeds, the changes bring about assimilation not by the incorporation of the forms or the information offered by the world but, instead, by a creative reshaping of the brain as well as the body that facilitates continuing interactions of the self with its world, insofar as that world is accessible to the brain and body. Learning to dance, to play the violin, or to play tennis (Dreyfus, 1979) requires changes in the body as extensive as those in the synapses of the brain.
The completion of the action-perception cycle up to and including assimilation is by incorporation of the new learning into the life history of the individual. Here is the stage where phenomena are experienced and consciousness appears in the cycle, long after the raw sense data, the phantasms, the flashing pinpoints of light, are gone. To be useful, each new experience must be integrated into the cumulative personal life history, by which actions are judged, new goals created, and new actions planned. In this view, consciousness comes only after an action has been initiated, not before, so that the action is perceived by the actor as a cause, and the sensory consequences as effects. This self-awareness of the intention-action-perceptionassimilation cycle is the basis for the concept of causation and for the critical importance of the time lag between effect and its necessarily prior cause. Studies by Piaget (1930) have shown that this association of cause and effect is laid down in the somatomotor phase of development, when an infant is learning to control its body. How and why the experience of awareness comes soon after in the way that happens are matters for speculation, but its phenomenology gives clear indication of both the powers and the limitations of this remarkable process. Its temporal range is enormous, but its momentary content is sparse. Our actions are influenced by our entire life history, but we are conscious of only minutes, intermittent fragments in sequential moments of thought. There is compelling clinical and physiological evidence that the medial temporal lobe is essential for the construction of the life history, which gives the wholeness of intentional structure, the self, personhood, or, more popularly, personality. However, that brain part is not necessary for consciousness, nor is the hippocampus a site of long-term memory storage. On the contrary, the dynamic processes that comprise intentionality in action are nonlocal, and likewise the personality to which it gives rise. Furthermore, most of intention is inaccessible to consciousness at any one moment, not so much that access is forbidden, rather that the sheer massiveness of the dynamic state space precludes squeezing the entire past through the eye of a needle. Reification of intentionality as 'the unconscious' appears to reduce this living body of experience inappropriately to a static warehouse of latent memories with locked doors.

In Platonic, Aristotelian, and Thomist doctrines a separation is made between the material and spiritual domains with identification of a spiritual agency, the soul, that moves the body. In the Cartesian metaphor, the soul is to the body as a pilot is to a ship. Most scientists today have adopted monist views that give no place to soul. Those adhering to a passive view of cognition find the source of agency instead in the genetic and environmental determinants of behavior, and debate the relative primacy of 'nature' $v s$ 'nurture'. Those practicing an active view propound the primacy of self-determination but find themselves uncomfortably skewered on the horns of the unresolved antinomy between 'free will' and 'determinism'. New developments in the sciences of nonlinear dynamics, complexity, and chaos indicate that this conflict is a pseudoproblem (Freeman, 2001). No human action is entirely determined by either genes, world or self but instead by an ever-shifting balance of interactions among the three formants. The challenge for neuroscientists is to 
analyze the brain dynamics by which goals emerge in the form of imagined future states, which lead to neural patterns of selective attention, preconfiguration of expected input, and actions that are intended to bring into conformance the flow of sensory input with the desired state. How do we come to dream of that which should be but is not, and then act to realize the dream? In principle, the material mechanisms by which novel patterns are created through nonlinear dynamics can explain the process, without resort to spiritual causes, although speculations are not thereby excluded that spiritual events may parallel material processes.

\section{THE NEURAL BASIS FOR THE CONSTRUCTION OF KNOWLEDGE THROUGH CULTURE}

Learning leads to assimilation, but assimilation by itself does not lead to knowledge, because knowledge is intrinsically social. It is embedded in the particular culture in and by which a group of humans live. Here is a question of profound importance for neuroscientists and neurophilosophers to answer. Given that assimilation is by the chaotic constructions within individuals that are unique in forms and contents to each individual, how can there be shared assimilation? The world is infinitely complex, offering a different face to each individual. No one can fully grasp any part in its fullness owing to the finite scope of our skills in comprehension. This is the reason we live our lives by continually posing hypotheses and asking questions that by their nature already contain the answers we get and nothing more. This is the reason that knowledge comes primarily by framing questions and only secondarily by finding the answers. The natural tendency of the individual in this on-going dialectic through learning is to become specialized and fade progressively away from humanity, ever deeper into an arcane discipline that can be shared with fewer and fewer aspirants to like understanding. Such is the loneliness of isolated graduate students and professionals in the humanities, which is comparable to the anomie of rootless urban drones, and the angst of intellectual dilettantes.

The natural sciences differ from the humanities in the respect that knowledge comes more overtly through joint action in the field and laboratory. Results of experiments from teams of scientists are communicated in scholarly conferences and publications with the intent of replication by other groups before acceptance. The most compelling agreement comes with useful application of new knowledge in the development of instruments, chemicals, procedures, and operations that change our ways of living, often dramatically, for better or worse. The more far-reaching developments lead to the institution of new courses of instruction, undergraduate majors, text books and monographs, academic departments, and entirely new industries with enormous economic and industrial investments involving the concerted actions of thousands, even millions, of workers. In brief, shared knowledge comes through shared action.

Assimilation through Hebbian learning is not enough to explain the neurobiological mechanisms by which understanding develops that bonds individuals by the process of socialization. Synaptic learning unchecked leads to isola- tion, lack of empathy, and inability to act in concert. Examples of extreme failures of socialization may include some autistic children and psychopaths who have astonishing cognitive skills but stunted emotional development. The neural mechanisms of learning with their tendency to isolation of the individual must be countered by opposing mechanisms. In the context of brain dynamics, the changes in brains that occur during learning under reinforcement are by the growth of self-organized intentional structure, which is progressively elaborated and differentiated. Learning of new structure that is logically inconsistent with existing structure in dynamical systems such as brains requires mechanisms for dissolution (also called 'unlearning', devolution or dedifferentiation-Freeman, 2001), which occurs by the induction of chaos prior to the emergence of new order.

There is abundant evidence for the existence and operation in the extremes of such mechanisms of chaotic dynamics in the widespread application of social technologies for behavioral modification involved in religious conversions, inculcation of political ideals and allegiances, indoctrination of recruits into military and paramilitary troops and teenage gangs, and the group bonding that occurs in fraternities, sororities, and large corporations. The changes in individuals brought about by use of these techniques do not involve forgetting or loss of memory, but instead occur by restructuring of their personalities. Dissolution presages new learning with deep, often dramatic, rarely catastrophic, changes in values and points of view that typically are life long. Coercive uses of the techniques are widely known as 'brainwashing' and 'reeducation'. These uses have had the unfortunate effect of obscuring the ubiquity and necessity of these techniques in normal socialization. A more felicitous appellation might be 'the Scrooge Effect', to emphasize the beneficial aspect of a night of terror. The dissolution prepares for new learning by self-organization, whereby the pre-existing life history of an individual is transiently weakened, even melted down, so that new structure can grow that is not logically consistent with all that has come before. The ubiquity of the process provides strong evidence, if any further is needed, that brains are dynamical systems and not logical devices.

The biological techniques for inducing dissolution are well known. Individuals separate themselves or are isolated from their normal social surroundings and support systems. They engage in or are subjected to severe physical exercise as in dancing, sports, and military drills, lack of sleep, chemical stresses of their brains through purgatives and fasting, and the induction of powerful emotional states of love, hate, fear, or anger. At some threshold the customary structure of the individual begin to crumble, and a collapse may occur that was described by Ivan Pavlov as 'transmarginal inhibition', the stage of physiological arousal beyond which further excitation leads to paradoxical depression. The experience may range from ecstatic visions of angels and blinding illumination through degrees of elation or discomfort to the stark terror of psychic free fall (Sargant, 1957). There is regression to successively earlier levels of assimilation as the structure of intentionality dissolves, particularly with resurfacing of old patterns of relations to parental care. There is a loss of normal constraints on 
behavior, and, in extreme instances, of language, locomotion, posture and even consciousness as the individual collapses. Recovery from collapse is followed by a state of extreme suggestibility, in which the skills of language and the competencies of daily living are regained, yet new values and habits can be established. This is carried out in a social setting of succor and loving care by attendants who induce by example and exhortation the cooperative behaviors that lead to shared beliefs and, above all, to blind trust in the new companions and the social organization they embody and provide. This is a two-way process, because the caregivers get strong feelings of satisfaction from their supportive actions, and the recipients have strong sensitivity to peer pressures experienced as feelings of need for approval. The process is frequently referred to as being reborn (Verger, 1954). In the absence of support there is reestablishment of the status quo ante, meaning that the opportunity for change can be lost, attesting to the high degree of dynamic stability that characterizes intentional structures in normal circumstances.

Little is known about the neurophysiology of these personal and social interactions, and even less about the neurochemistry of the changes in brains that occur in and are induced by dissolution. The evolutionary antecedents of the techniques for socialization are likely to be found in the processes by which bonding occurs in altricial mammals as a necessary basis for reproduction (Freeman, 1995). The most likely candidate for a leading role in dissolution is a chemical neuromodulator named oxytocin (Pedersen $e t$ al, 1992). This neuropeptide has been known for many years as the agent in the female body that induces labor in parturition and subsequently lactation in the nourishment of the young. More recently, oxytocin has been found to be released by the brain into itself during sexual intercourse, particularly during orgasm in both men and women, and to be implicated in pair bonding not only of the parents to the child but also of parents to each other. The neurochemical actions of oxytocin in the brain are widespread, extremely complex, and difficult to study, so that much remains to be explored, but present knowledge shows that this neuropeptide is capable of inducing the meltdown of past learning that enables new learning. A simple example is the release of oxytocin flooding the brain of the multiparous ewe during delivery of her second and later litters, following which the dam refuses to nurse her earlier litters, having expunged the olfactory imprint required for maternal recognition of them as her offspring (Kendrick et al, 1992). This primitive but well-documented instance of dissolution serves also to explain its biological utility. Oxytocin is not likely to act alone, rather in concert with other neuropeptides, the neuroamines, and an array of amino acids from the brain stem nuclei and periaqueductal gray matter, all known to mediate states of emotion and levels of affect and disposition (Panksepp, 1998; Pert, 1997). However, existing data and theory are alike inadequate to the task of modeling neurochemical systems of this complexity.

I postulate that affiliation (Carter et al, 1997) is realized through new learning by cooperative behaviors driven by brains that have been prepared by the neurochemical changes precipitating dissolution, a chaotic state transition that leads to regression and clears the way to the formation of new brain circuits. Cooperative action is the bedrock of social bonding for the same reason that brains work by creating and testing hypotheses as their means for information processing. The reason is that each individual in a social group is infinitely complex and can never be known completely by any other individual. The limitation is ever more severe as the size of groups grows larger than the nuclear family. Inadequacy of knowledge is compensated by the development of blind trust, which transcends language and provides unquestioning life-long bonds and allegiances. The social technology of bonding is well known, having been explored by anthropologists in studies of tribal rites of passage, ordeals, and ceremonies (Verger, 1954), invariably accompanied by use of music, drumming, dance, and other forms of predictable repetitive actions, and by symbols such as flags, icons, totems, and, in modern times, corporate logos, military insignias, and the colored armbands of teenage gangs. I suggest further that the examples from the conversions cited above may be extremes, and that dissolution may be occurring episodically throughout infancy and childhood, and perhaps in minimal degree every night during sleep and dreaming. The process clearly deserves more attention and study than has yet been forthcoming.

\section{INFERENCES REGARDING PSYCHIATRIC PRACTICE}

The human brain is finite, whereas the world is infinite in complexity and variety. We learn what we need and what we can by assimilation, which is more than adaptation by acquiescence. Assimilation follows active intrusion into the parts of the world that are accessible to us, shaping our bodies and brains, and the world, to meet our expectations, projections, desires, and dreams. Our cumulative experience, when shared with others of our kind through joint actions, supports the growth of knowledge. In the fields of politics and of science, which is a vast transnational social enterprise, knowledge takes the form of 'laws'. These laws are not eternal truths: they are tools we use to predict, plan, act, and test hypotheses. An example is the law of causality by which we build chains of cause and effect through time. The concept is essential for the assignment by parents, teachers, courts, and tribunals of credit and blame, reward and punishment. This same tool is also widely used in science and technology for constructing linear chains of causes in search of convincing evidence, often referred to as 'the smoking gun'. This approach gives a feeling of necessary connection, but it is out of place in therapeutic situations, where the task is to determine risk factors, predictors, and relations in order to fix the problem and not the blame. A prominent example of misuse was the claim by cigarette makers that there was no proof of a causal connection between smoking and lung cancer. That claim impeded efforts of public health officials to educate the public on the dangers of smoking.

Mental health workers are charged with the responsibility for assisting those who are distressed or who afflict others with their unacceptable experiences and behaviors. Their aim is to bring about changes in attitudes and behaviors through learning. Not surprisingly their therapeutic approaches, as they have evolved over many centuries, have relied on the well-known techniques of both individual and social assimilation in varying degree and emphasis. Many 
Western psychiatrists invest heavily in cognitive methods of behavioral modification of individuals by teaching, analyzing, explaining, exhorting, and, in extreme cases, coercing conformity within accepted standards of the community. Others rely heavily on mobilization of community support, especially from family and friends (Frankl, 1973). Drugs are admissible, even required by practice or law, usually though not always with the consent of the subject. The use of electroconvulsive therapy (ECT) may be likened to the use of drugs, in providing a shortcut to force dissolution and regression physically, so as to induce a state of malleability and an opportunity for re-education, although with a heavy price in disruption of memory. We may infer that the desired changes in brain dynamics of learning take place through Hebbian association and anti-Hebbian habituation, extinction, and normalization, which are enacted and supported by neurohumoral agents, including the biogenic amines and neuropeptides in collaboration with nerve growth factors that operate by mobilizing sections of the genome (Panksepp, 1998; Pert, 1997).

Faith healers and shamans in technologically less complex social groupings rely on techniques that many westerners regard pejoratively, and shortsightedly, as brainwashing. There is heavy emphasis on the familial and social contexts to which behaviors are to be adapted, and there is strong reliance on altered states of consciousness involving the induction of strong emotional arousal leading to trance, transmarginal inhibition, and hallucinatory experiences. The use of drugs such as alcohol, nicotine, mescaline, ayahuasca, and peyote might be regarded as shortcuts to avoid dependence on arduous and prolonged practices such as fasting, purging, vigil keeping, withdrawal through meditation, and the suffering of painful stimuli, with the possibility, often intentional, of mutilation and scarification in rites of passage. As noted already, the neurochemistry and synaptic changes underlying these often dramatic modifications of behaviors are still largely unexplored by neuroscientists, owing to the extreme circumstances in which they come into play.

Psychoanalysis has different mixes of many of these components. In contrast to most other approaches the relation is conventionally dyadic between the therapist and the patient. The use of drugs seems not to be a part of the culture, perhaps because of early adverse experience with cocaine, and ECT is eschewed. The classic approach using free association may be likened to the unsupervised learning used in recurrent neural networks, the blind search for unknown forms using techniques that are described as random walks, Markov processes, and genetic algorithms. These are the techniques used by bacteria and genes that have no brains. The use of the method in psychoanalysis is based on the premise that the structure generated by intentionality is illogical, vast, and dynamically partitioned into loosely interconnected domains, some of which may have been walled off by dynamic barriers as an adaptive mechanism for the avoidance of psychic pain. The aim of the analysts to assist a patient to rove freely through this mine field of adverse associations in the hope of detecting signs of emotional arousal that reflect proximity to a guarded domain of traumatic memories, that is exerting malign influence on rational behavior without the awareness of the patient. If the dynamic barrier can be surmounted, then that forbidden domain might be brought to consciousness for assimilation and resolution of conflict.

Prior to an attempt to use neurodynamics to gain insights into the therapeutic process, let it be acknowledged that the goal of constructing a map or thesaurus between concepts in neurodynamics and psychoanalysis is illusory. For an example in a nearby field, an impasse has been reached in attempting to map 'genes' into 'DNA sequences'. A geneticist, having localized a gene for a trait like eye color, cannot get a straight answer from a molecular biologist as to which particular sequence of the four base pairs of nucleic acids constitutes the gene. The intellectual structures of the knowledge bases in classical and molecular genetics have deep roots in cultural domains that are too far apart to allow any simple translation between them. Yet the disciplines address the same phenomena from differing perspectives and can be expected to complement one another. Similarly, in physics a photon may be a wave, a particle, both, or neither, depending on the context. Here the neuroscientific structure of 'microscopic-mesoscopic-macroscopic-neurodynamics' is comparable to the philosophical structure of 'intention-action-perception-assimilation' and to the psychoanalytic structure of 'id-ego-superego-transference', in that these concepts all address the same subject. The problem at issue is not how to map each term into the others, but to extract insights into each domain from distinctions that are obvious, even inevitable, only in the others.

Neurodynamics, intentionality, and psychodynamics are descriptions of processes by which structures are created in brain state space, character, and personality. The systems all postulate an imbalance of some kind as the genesis of action, variously described as a nonequilibrium chemical process like a deficit of glucose, as a need like thirst, curiosity, or hunger for power ('drive'), as an intent constituting the prediction of a future state ('stretching forth'), and as the id ('it'). They all postulate goal orientation but with differences such that with intent the goals are internally derived in each subject, whereas with drive the goals are externally and universally defined by observers, and with id there is an appeal to genetic transmission of urges in mythic proportions. Cognitive structures are founded on these bases. Reasons, purposes, and motives are the explanations given for actions taken or observed. Wishes, desires, and dreams are the phenomenological experiences of actions contemplated or engaged. Hormone, will, eros, and libido are causal agencies relating to actions directed toward homeostasis, sexual pleasure, reproduction, love, companionship, hatred, and power. Such lists do little more than sketch the complexity of human affairs. Where the problem comes into focus is in the application of theory to understand the dynamics of mental disorders, with the intent of changing the intentional structures of disturbed individuals. Of many possible insights and perspectives, the most important concern the phenomena of assimilation, dissolution, and new learning that is possible, after the process of chaotic destabilization has led to regression and a clean slate, or some degree thereof.

The concept of transference clearly relates to the dynamics of assimilation, whether to objects, events, situations, or other persons, but particularly between the 
patient and the therapist. Neurodynamics may illuminate the process by enabling the distinction between structural advancement by conventional, constructive learning in Hebbian association, habituation and normalization vs advancement through discontinuous and illogical modification by chaotic dissolution and rebuilding in new directions. The distinction is more than a matter of degree of change. At one pole is the cool detachment of cognitive therapy, which is appropriate, for example, in depression owing to chemical imbalance, bereavement, guilt, or shame. At the other pole is fierce emotional involvement in abreactive techniques, which are appropriate, for example, in post-traumatic stress disorder, dissociative identity disorders, and various types of neurotic maladaptation. Neurodynamics helps to show that assimilation and dissolution may be active at both extremes and in varying degrees between the poles, while emphasizing that two very different kinds of chemistry, neural processing, and therapeutic methodologies are at work, whether simultaneously or sequentially.

An essential element to breaching the resistance in a patient to confrontation of buried material is postulated to be an intense emotional reaction that may be comparable to the prerequisite for a conversion experience. This insight implies that an approach to understanding the biology of abreaction and the malleability that follows may require the same neuroscientific exploration of brain dynamics that will be needed to understand the neural mechanisms of social group formation. However, strong emotion by itself is insufficient. Every patient comes to a therapist after making an intentional decision, which carries an expectation and hope of relief to be found through guidance and benefaction. Likewise, the therapist accepts a new patient with the hope of a perfect match. Both are inevitably disappointed in varying degrees as analysis progresses, arousing perhaps quite strong hope, joy, frustration, anger, nostalgia, and fear, indeed at different times the entire array of human emotions. What may yet be lacking is perception by the patient of his or her own conflicts and inconsistencies in intentional structure. Perception of them may be the key to destabilization as a prelude to dissolution. Clearly, this perception carries the weight of danger to the patient and to the therapeutic relation. The analyst may also experience fear, and back away from the prospect of the collapse of intentionality, which may be a strong medicine indeed.

Here is the cusp of choice. Many analyses, perhaps most, careen through misunderstandings, false leads, open wounds, short-term insights, and small triumphs, but peter out with no clear resolution, or, even more disheartening, with the patients' children turning up on the analyst's couch, bringing the same complaints as their parents. There may be no ignition of an intense encounter, no warning sign of incipient destabilization to alert the analyst to a weakness in the intentional structure that has been jangled, no manifestation in tremors of the hands and lips, evasiveness of the eyes, breaks in the voice and train of thought, restless changes in posture, even pacing, but indecisively. If and when these signs appear, the analyst needs a sure grasp of personality dynamics to decide whether to cut and run, or to seek confrontation and precipitate collapse, and be ready to solicit change in a new direction, having already thought through what that direction might best be, based on deep knowledge of the history, family, physiological status, and intentional structure of the patient, and be ready to give handsomely of time, energy, and emotional support during the transition.

\section{CONCLUSIONS}

The convenience, utility, and attractiveness of concepts from nonlinear dynamics in advancing further studies of brain functions and therapeutic relations is obvious, so that some words of caution may be in order. Dynamics in engineering is well grounded in the science of measurement, so that the numbers coming from observations on physical and economic systems can be used to test theories that are expressed in probability distributions and differential equations. Without the numbers the theories are metaphors. Without suitable yardsticks and spaces there are no numbers. The Shannon-Weaver theory provides a superb yardstick for the measurement of information but does so by divorcing information from meaning. There is no yardstick for meaning, nor is it likely that there can ever be. The use of metaphors from dynamics for heuristic purposes in psychiatry and psychoanalysis to complement and inform the classical concepts and myths is to be commended, provided that they are not taken too literally. The only existing yardstick of a proposed brain theory is the extent to which theory improves the statistics of the numbers of people who have been compromised in their social and personal lives by mental disorders, the numbers of clients who have been restored to competency by treatment regimens, and the economic and social costs of doing so. Even these numbers are hard to come by, but they are the best yardstick we now have, and if brain theory suffices to emphasize this aspect, in addition to explaining the necessity for the dissolution of intentional structure prior to the creation of fundamentally new structure, theory will have paid its way.

\section{ACKNOWLEDGEMENTS}

This research was supported by a Grant MH 06686 from the National Institute of Mental Health. This essay was adapted from a lecture given at a symposium on 'Foundations and the Ontological Quest: Prospects for the New Millennium' in Rome, Italy 7-10 January 2002.

\section{REFERENCES}

Aquinas St. Thomas (1272/1952). The Summa Theologica. Translated by Fathers of the English Dominican Province. Revised by Daniel J Sullivan. Published by William Benton as Volume 19, Great Books Series. Encyclopedia Britannica, Inc.: Chicago.

Carter CS, Lederhendler II, Kirkpatrick B (1997). The integrative neurobiology of affiliation. Ann NY Acad Sci 807: 501-503.

Dreyfus HL (1979). What Computers Can't Do: The Limits of Artificial Intelligence. Harper Colophon: New York.

Frankl V (1973). The Doctor and the Soul. Random House: New York.

Freeman WJ (1995). Societies of Brains. Lawrence Erlbaum Assoc.: Mahwah, NJ.

Freeman WJ (2000). Neurodynamics. An Exploration of Mesoscopic Brain Dynamics. Springer-Verlag: London, UK. 
Freeman WJ (2001). How Brains Make up Their Minds. Columbia University Press: New York.

Freud S (1895). The project of a scientific psychology. In: M Bonaparte, A Freud, E Kris (eds). The Origins of Psychoanalysis, (E Mosbacher \& J Strachey, Trans (1954)). Basic Books: New York. pp 356-359.

Geschwind N, Kaplan E (1962). A human cerebral deconnection syndrome - a preliminary report. Neurology 12: 675-685.

Gibson JJ (1979). The Ecological Approach to Visual Perception. Houghton Mifflin: Boston.

Glass L, Mackey MC (1988). From Clocks to Chaos: The Rhythms of Life. Princeton University Press: Princeton, NJ.

Kendrick KM, Levy F, Keverne EWB (1992). Changes in the sensory processing of olfactory signals induced by birth in sheep. Science 256: 833-836.

Merleau-Ponty M (1945/1962). Phenomenology of Perception, (C Smith, Trans.). Humanities Press: New York.

Panksepp J (1998). Affective Neuroscience: The Foundations of Human and Animal Emotions. Oxford University Press: Oxford, UK.
Pedersen CA, Caldwell JD, Jirikowski GF, Insel TR (eds) (1992). Oxytocin in maternal, sexual, and social behaviors. Ann NY Acad Sci 652: 194-211.

Pert CB (1997). Molecules of Emotion: Why You Feel the Way you Feel. Scribner: New York.

Piaget J (1930). The Child's Conception of Physical Causality. Harcourt, Brace: New York.

Ohl FW, Scheich H, Freeman WJ (2001). Change in pattern of ongoing cortical activity with auditory category learning. Nature 412: 733-736.

Sargant WW (1957). Battle for the Mind. Greenwood: Westport, CT.

Skinner BF (1969). Contingencies of Reinforcement: A Theoretical Analysis. Appleton-Century-Crofts: New York.

Sperry RW (1950). Neural basis of the spontaneous optokinetic response. J Comp Physiol 43: 482-489.

Verger P (1954). Dieux d'Afrique. P Hartmann: Paris. 